\title{
Errata
}

(Proc. Indian Acad. Sci. (Math. Sci.), Vol. 117, No. 1, February 2007, pp. 49-59)

\section{On the problem of isometry of a hypersurface preserving mean curvature}

HÜLYA BAĞDATLI and ZIYA SOYUÇOK

Reference to equations in Theorem 1 (Fundamental theorem) should be eqs (5.1), (5.2), (5.3) and (5.4) instead of eqs (4.21), (4.22), (4.23) and (4.24).

(Proc. Indian Acad. Sci. (Math. Sci.), Vol. 113, No. 1, February 2003, pp. 15-51)

\section{The planar algebra associated to a Kac algebra}

VIJAY KODIYALAM, ZEPH LANDAU and V S SUNDER

Definition 4.9(c) is incorrect; it should be changed so as to read:

(c) The action $\alpha$ is called outer if $M^{\prime} \cap\left(M \rtimes_{\alpha} H\right)=\mathcal{C}$.

And part (f) of Proposition 4.10 should hence be omitted.

Fortunately, this oversight has no unpleasant consequences for the results in this paper due to the fact that this (new) condition implies the earlier (erroneously stated) condition. The implication stated in the last sentence is a consequence of Theorem 4.11, whose proof, in turn, uses only the (proposed new) form of the condition that is stated above. 
Errata (Continued)

(Proc. Indian Acad. Sci. (Math. Sci.), Vol. 115, No. 3, August 2005, pp. 279-307)

\title{
On degree-regular triangulations of torus and Klein bottle
}

\author{
ASHISH KUMAR UPADHYAY*
}

The article [1] appeared in pages of this journal. This note is a correction to a minor error in Example 2 on page 285 of the article. Though this error has no effect on results and content of other parts of the article, it may lead to serious errors if the expression is used without correction. In the referred example, a series of weakly regular orientable combinatorial 2-manifolds on $2 n$ vertices, of Euler characteristic 0, are given as:

$$
T_{n, 2, k}=\left\{u_{i} u_{i+1} v_{i+1}, u_{i} v_{i} v_{i+1}, u_{i+k} u_{i+k+1} v_{i}, u_{i+k+1} v_{i} v_{i+1}: 1 \leq i \leq n\right\},
$$

where $n \geq 4$ and $k=1, \ldots,(n-3)$, addition in the subscripts being modulo $n$.

In the text thereafter, $1 \mathrm{k}\left(u_{i}\right)$ is presented as " $\operatorname{lk}\left(u_{i}\right)=C_{6}\left(u_{i-1}, v_{i}, v_{i+1}, u_{i+1}, v_{n+i-k-1}\right.$, $\left.v_{n+i-k}\right)$ ". If we substitute $n=6, k=1$ and $i=5$ in this expression, we get $\operatorname{lk}\left(u_{5}\right)=C_{6}\left(u_{4}, v_{5}, v_{6}, u_{6}, v_{3}, v_{4}\right)$, whereas, in fact, it should have been $\operatorname{lk}\left(u_{5}\right)=$ $C_{6}\left(u_{4}, v_{5}, v_{6}, u_{6}, v_{4}, v_{3}\right)$ as is also evident by the picture below:

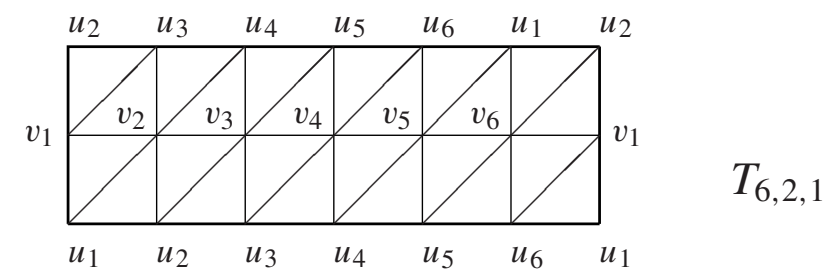

Now, using the expression for $T_{n, 2, k}$ one gets the corrected link of $u_{i}$ as: $\operatorname{lk}\left(u_{i}\right)=$ $C_{6}\left(u_{i-1}, v_{i}, v_{i+1}, u_{i+1}, v_{n+i-k}, v_{n+i-k-1}\right)$.

\section{References}

[1] Datta B and Upadhyay A K, Degree regular triangulations of torus and Klein bottle, Proc. Indian Acad. Sci. (Math. Sci.) 115(3) (2005) 279-307

\footnotetext{
*The author is now with School of Basic and Applied Sciences, Guru Gobind Singh Indraprastha University, Kashmere Gate, Delhi 110 403, India.

E-mail: akupadhyay@ipu.edu
} 